\title{
The DLR Lightweight Robot - Design and Control Concepts for Robots in Human Environments
}

\author{
A. Albu-Schäffer, S. Haddadin, Ch. Ott, A. Stemmer, T. Wimböck, and G. Hirzinger \\ Institute of Robotics and Mechatronics, German Aerospace Center (DLR), Germany \\ E-mail: alin.albu-schaeffer@dlr.de, sami.haddadin@dlr.de, christian.ott@dlr.de, \\ andreas.stemmer@dlr.de, thomas.wimboeck@dlr.de, gerd.hirzinger@dlr.de
}

\begin{abstract}
The paper presents a new generation of torque-controlled lightweight robots (LWR) developed at the Institute of Robotics and Mechatronics of the German Aerospace Center. In order to act in unstructured environments and interact with humans, the robots have design features and control/software functionalities which distinguish them from classical robots, such as: load-to-weight ratio of $1: 1$, torque sensing in the joints, active vibration damping, sensitive collision detection, as well as compliant control on joint and Cartesian level. Due to the partially unknown properties of the environment, robustness of planning and control with respect to environmental variations is crucial. After briefly describing the main hardware features, the paper focuses on showing how joint torque sensing (as a main feature of the robot) is consequently used for achieving the above mentioned performance, safety, and robustness properties.
\end{abstract}

\section{INTRODUCTION}

The DLR lightweight robots have been developed for application areas which are fundamentally different from the ones of classical industrial robotics. The strengths of industrial robots are especially high positioning accuracy (repeatability and absolute accuracy), high speed, durability, and robustness, as well as the relatively low price. Therefore, today's industrial robots are used especially in well structured environments, in which the position and shape of the parts to be manipulated are well determined and in which collisions with the environment and humans can be estimated and excluded in advance. High performance is achieved for fast tasks which are repeated numerous times. Generally, the high positioning accuracy requires high stiffness at the price of high robot mass relative to its payload.

In contrast, the robotic systems developed at DLR (arms, hands, a humanoid manipulator) are designed for interaction with humans in unstructured, everyday environments. In such applications, high absolute positioning accuracy cannot be exploited due to limited accuracy of position information about the surrounding environment, while its side-effects in design (high stiffness and mass) are clearly undesired. The DLR robots (Fig. 1) are thus designed for application areas which are generally not covered by industrial robots, but are still ongoing research topics. Typical examples are:

- Assembly processes for which the position estimation for the mating parts and/or the positioning accuracy of the robot is significantly below the assembly tolerance.

- Applications in which the robot works in immediate vicinity of humans and possibly in direct physical cooperation with them.

- Mobile service robotics applications (arms mounted on mobile platforms), for which the information about the position of the robot and the surrounding objects, as well as about the dimension of these objects is afflicted with relatively high uncertainty.

For the mechanical design, the mentioned applications determine the requirement of a low robot mass compared to the payload in order to enable mobility and minimize the injury risk. However, the robots are operated at relatively low velocities compared to industrial robots, thus enabling higher gear ratios. The main requirements for the electronic design result from the high number of sensors, such as joint torque sensors, redundant position sensing, and wrist force-torque sensing. Furthermore, motor 


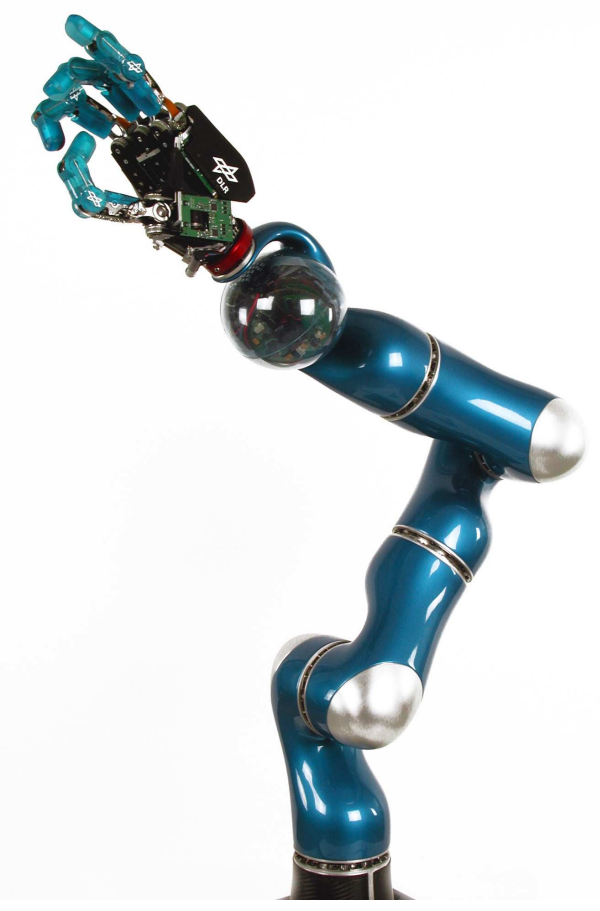

Fig. 1. The DLR lightweight robot arm and hand - a new generation of robots.

and sensor electronics have to be integrated to reduce the number of wires in the arm. This in turn requires a fast and deterministic bus communication between the joints in order to be able to implement control algorithms on a central computer.

Within this new robot concept, a strong emphasis is set on the design of control laws which can provide robust performance (with respect to positioning and model uncertainty), as well as active safety for the human and the robot during their interaction. Compared to standard industrial robot control, the following aspects are of particular importance:

- Position control has to compensate the effects of the robot elasticity (such as vibrations or the steady state position displacement) to ensure the performance of positioning and trajectory tracking. This problem exists (although in a reduced amount) also for industrial robots moving at high velocities. In the control of the DLR robots, the torque sensing in each joint plays a key role. These sensors constitute an essential feature compared to most other robots: they measure the joint vibration behind the gear-box and therefore enable an active vibration damping. Taking into account the elasticity of the transmission, each joint becomes a mass-spring-damper system (and thus a fourth order system), so that the complete state is given by position and velocity (as for the second order rigid robot model), and additionally by the torque and its derivative. Thus, measuring the torque is essential for implementing full state feedback control laws.

- The most important feature in the control of the DLR robots, however, is the use of the joint torque sensors for so-called soft robotics control, i.e. compliance and force/torque control, as well as for collision and failure detection.

In Sec. II the paper will give a short overview of the LWR hardware components. Sec. III and IV explain the above mentioned soft robotics control strategies and finally some example applications are shown.

\section{HARDWARE OVERVIEW}

The main design goals of the DLR lightweight robots were to build a manipulator with kinematic redundancy similar to the human arm, i.e. with seven degrees of freedom (DOF), a load-to-weight 
ratio of approximately 1:1 (industrial robots typically have a ratio of 1:10 or lower), a total system weight of less than $15 \mathrm{~kg}$ for arms with a work space of up to $1.5 \mathrm{~m}$, and a high dynamic performance. There should be no bulky wiring on the robot and no electronics cabinet as usually required by typical industrial robots.

The full state measurement in all joints is performed in a $3 \mathrm{kHz}$ cycle, using

- strain gauge-based torque-sensing,

- motor position sensing based on magneto-resistive encoders, and

- link-side position sensing based on potentiometers (used only as redundant sensors for safety considerations).

The torque sensors are mounted on the flex spline component of the Harmonic Drive gear and therefore measure the joint torques acting on the links, while an additional bearing decouples the disturbing forces and torques. The sensor error is below $0.5 \%$ and a low-pass filtering at $600 \mathrm{~Hz}$ is used.

The robot joints are serially connected with the central computer via an optical fiber bus system, using the standardized real-time SERCOS protocol. The desired and actual motor position, the link torque and the link position are transmitted once every millisecond. Status and supervisory signals are transmitted by means of an acyclic channel.

The joint level control is implemented at $3 \mathrm{kHz}$ rate on a signal processor in each joint, while the robot dynamics and the Cartesian control are computed in a $1 \mathrm{kHz}$ cycle on a central computer.

Only five wires inside the arm are needed in order to connect all joints with the power supply and the external controller PC. The power consumption of the whole arm in normal operation is below $150 \mathrm{~W}$, thus by an order of magnitude lower than that of a comparable industrial arm. Still the arm is capable to handle $15 \mathrm{~kg}$ at low velocity, i.e. more than its own weight $(14 \mathrm{~kg})$. This high performance is achieved by using lightweight Harmonic Drive gears and the new RoboDrive motors possessing high energy density. These motors have been particularly optimized for service robotics applications where speed is relatively low, but minimal weight and power loss are crucial. Furthermore, the motors provide high dynamics during permanently reversing operation around zero speed.

The robot arm concept aimed at a completely modular assembly system with only a few basic components concerning joint mechanics, electronics, and links, so that different configurations can be composed in a short time (Fig. 2).

\section{Control Aspects for Robots Acting in Human ENVIRONMENTS}

This section will focus on the specific control aspects determined by this new hardware design and the operating conditions close to humans in unstructured environments.

As already mentioned in the introduction, torque sensing and feedback becomes essential, both for increasing motion accuracy of the flexible arm, as well as for direct monitoring and control of the interaction forces.

Measuring the torques in the joints is important, since the robot is always likely to collide or deliberately be in contact with its surrounding environment. These collisions, which may occur along the entire arm structure, can be directly detected using the torque sensors. For enhancing the precision of the tip force control, an additional wrist force-torque sensor can be used if necessary. Another advantage of using joint torque sensing is the fact that they are placed close to the actuators. This "collocation" of actuators and sensors is advantageous from a control point of view, enabling robust, passivity based control approaches.

The preference for passivity based control is another consequence of the fact that the mechanical properties of the manipulated objects and of the contacted environment are not known precisely. All controllers have intuitive physical interpretations related to passive mechanical elements, such as virtual inertias or multi-dimensional springs and dampers. Thus, the amount of energy introduced into the system by the controller is directly monitored. Therefore, stability can be ensured in contact with any environment, as long as it displays a passive behavior as well. 


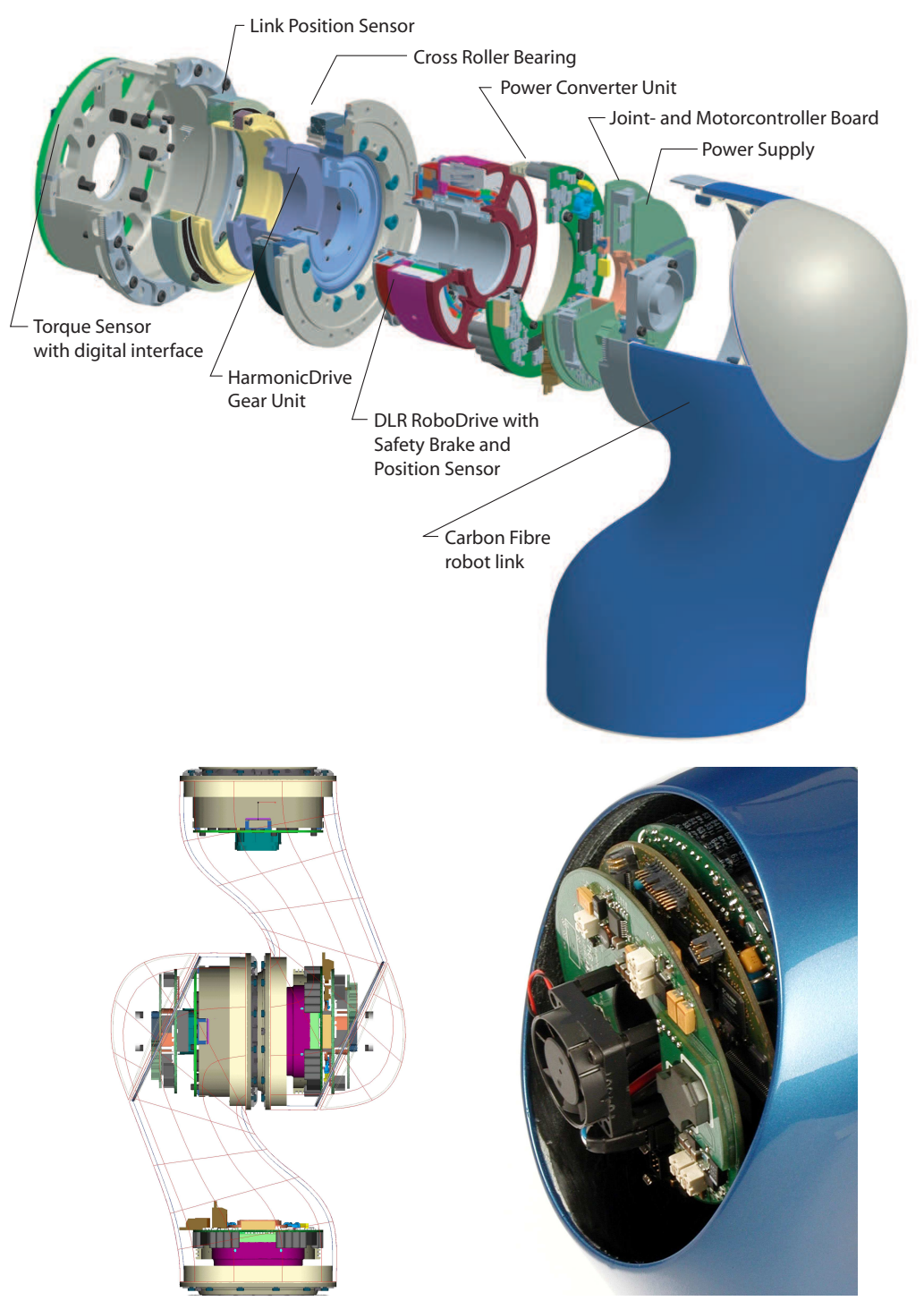

Fig. 2. The mechatronic joint design of the LWR including actuation, electronics, and sensing.

The next sections will exemplify how this approach is consequently followed starting with basic functionalities such as joint control or collision detection/reaction and leading to application oriented controllers for industrial assembly or to humanoid task level control.

\section{A. Joint Level Control}

At joint level, a decentralized state feedback controller (see Fig. 3) is implemented by using the entire joint state in the feedback loop, namely the motor position and velocity $(\theta, \dot{\theta})$ as well as the joint torque and its derivative $(\tau, \dot{\tau})[1]$. An alternative to the joint torque is to use the link side position and velocity for control. In practice, it is however very difficult to achieve a resolution and a precision of this position measurement which enables a useful estimation of the elastic deformation (and thus of the torque) between motor and link position.

By appropriate scaling of the feedback gains, the controller structure is used to implement position, torque or impedance control. For example, high torque and torque derivative gains, zero position gain and positive velocity feedback (for compensation of viscous friction) provide a torque controller, while high position and velocity gains are used for position control, together with lower torque feedback gains for vibration damping. On the other side, the desired torque for the controller is commanded according 


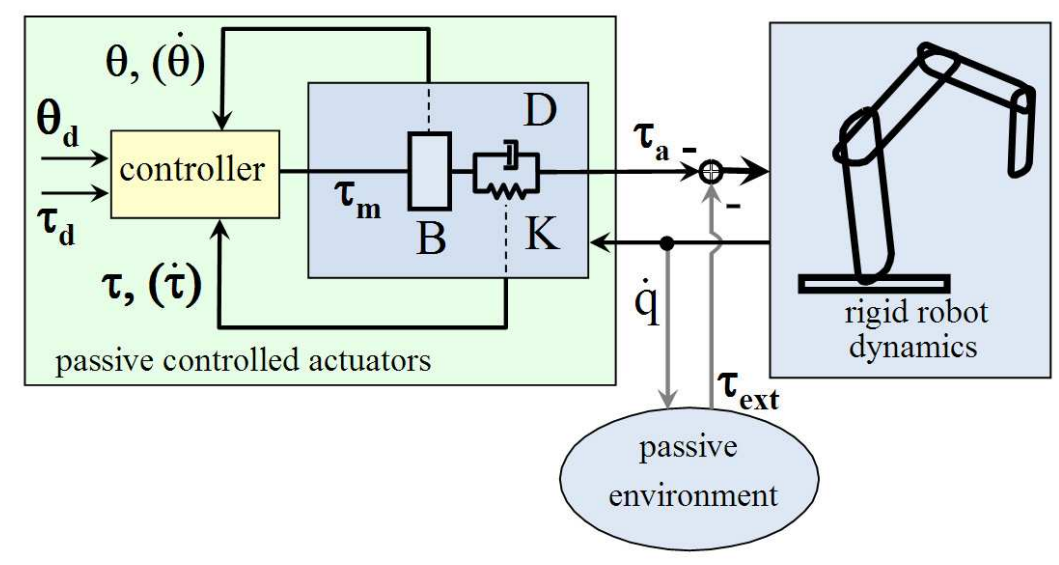

Fig. 3. Structure of joint level controller.

to the expected robot dynamics (e.g., if the robot is not moving, this corresponds to the gravity torques). Therefore, the robot operates in the so called "zero gravity mode", in which the motors compensate the robot's own weight. In this mode the robot can easily be moved by a human in order to teach trajectories or avoid injuries in case of collisions.

The use of the torque signal for vibration damping is illustrated in Fig. 4. Therein, a simple PD controller (with feedback of $\theta$ and $\dot{\theta}$ ) is compared to a state feedback controller, in which torque and torque derivative are used additionally. In Fig. 4a, the PD gains of both controllers are identical. While the response of the pure PD controller exhibits strong oscillations, the state feedback controller is well damped, but somewhat slower ${ }^{1}$. In Fig. 4b, the position feedback for the PD controller is decreased in order to achieve the same link side stiffness as for the state feedback controller. The response times of both controllers in Fig. 4b are therefore similar, but for the PD controller the position error is considerably larger and the oscillations are still present on the torque signal at the end of the trajectory.

The feedback terms turn out to have very intuitive physical interpretations: torque feedback reduces the apparent inertia of the motors, as well as the joint friction. Motor position feedback is equivalent to a physical spring while velocity feedback produces energy dissipation (viscous friction). These interpretations allow the passivity (and thus stability) analysis mentioned at the beginning of this section and enable also a consistent generalization to Cartesian impedance control.

\section{B. Cartesian Impedance Control}

During applications in which the robot is mainly in contact with the environment (e.g. automatic assembly), it is useful to control the forces rather than the positions in some Cartesian directions. A smooth transition between both operation types is realized by impedance control, where, rather than controlling generalized force or position, the relation between them is specified (e.g. as a stiffness and damping) together with a nominal desired position. With the physical interpretation of torque and position feedback given in the previous section, it is intuitive to design a Cartesian compliance by utilizing the joint level torque controller for reduction of motor inertia and friction and by replacing the joint level stiffness with a Cartesian spatial spring [1], [2], [3]. For implementation of this virtual spring, the motor position has to be used again, in order to preserve passivity. However, the desired tip position and the stiffness is specified in terms of link position $\boldsymbol{q}$. Therefore, a (statically equivalent) estimate $\overline{\boldsymbol{q}}(\boldsymbol{\theta})$ for $\boldsymbol{q}$ is computed, based only on the motor position $\boldsymbol{\theta}$ and the joint stiffness. The forward kinematics $\boldsymbol{x}(\overline{\boldsymbol{q}})$, the Jacobian $\boldsymbol{J}(\overline{\boldsymbol{q}})$, gravity torques $\boldsymbol{g}(\overline{\boldsymbol{q}})$, and the impedance law are computed based on this value, leading to a passive structure as shown in Fig. 5. Desired contact forces can be specified

\footnotetext{
${ }^{1}$ The torque feedback reduces the stiffness seen from the link side and therefore increases the response time.
} 

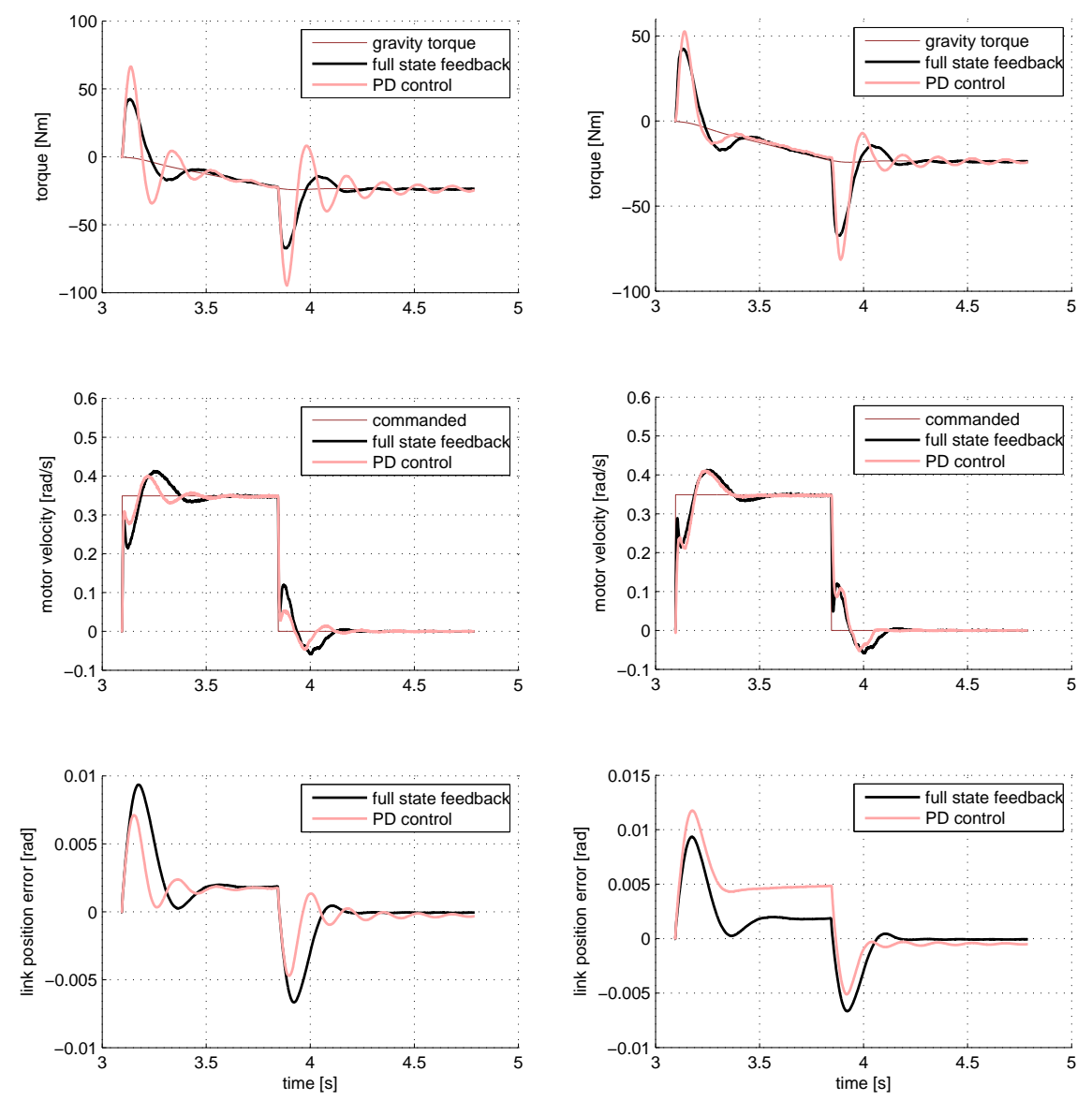

b)

Fig. 4. PD versus state feedback control. Left (a): the gains of the PD controller are identical to the position and velocity feedback gains of the state feedback controller. The PD controlled robot exhibits oscillations. Right (b): The gains of the PD controller are reduced, such that both controllers have the same link side stiffness. The PD controller has higher position errors and still some oscillations on the torque signal.

either indirectly by commanding a desired position and stiffness, or directly by transforming the desired force trough the transposed Jacobian to a desired joint torque.

Singularities of the Jacobian matrix clearly represent an obstacle to the implementation of a Cartesian impedance in some joint configurations. Therefore, we compute the well-known kinematic manipulability measure and use it as an optimization criterion for the robot's nullspace motion. In case that the singularity, however, cannot be avoided by a pure nullspace motion, we additionally use its differential for implementing a repelling force field. While this force field also affects the Cartesian impedance behavior, it ensures that singular configurations are avoided.

\section{Inverse Kinematics}

In contrast to the commonly used well-known Moore-Penrose pseudoinverse, a constraint optimization algorithm was chosen and investigated for dealing with the inverse kinematics problem of the redundant LWR [4]. An algorithm was developed which allows the introduction of constraints at the kinematics level. With this constraint optimization approach, singularity handling is realized, in order to enable the crossing of singularities along a specified path. For singularity crossing, two different strategies are known: deviation from the desired trajectory and deceleration from the desired trajectory. Within the 


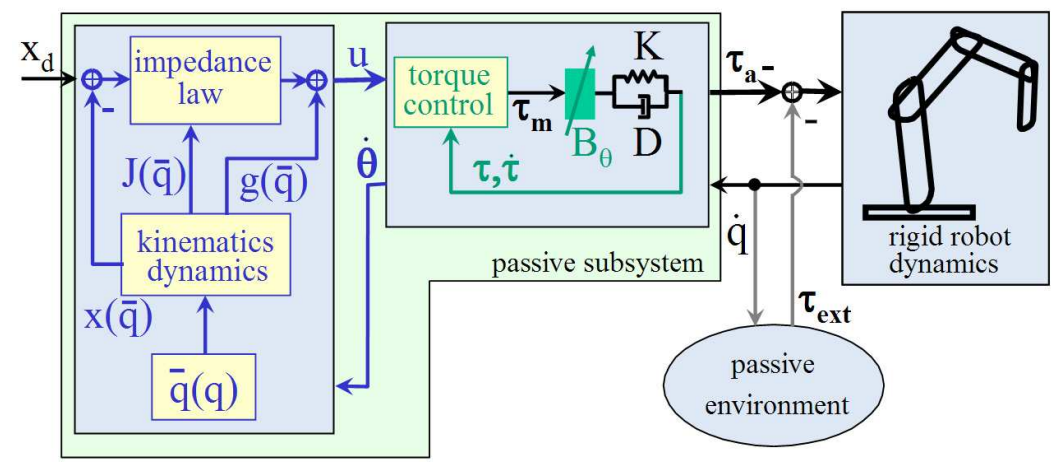

Fig. 5. Structure of Cartesian impedance controller.
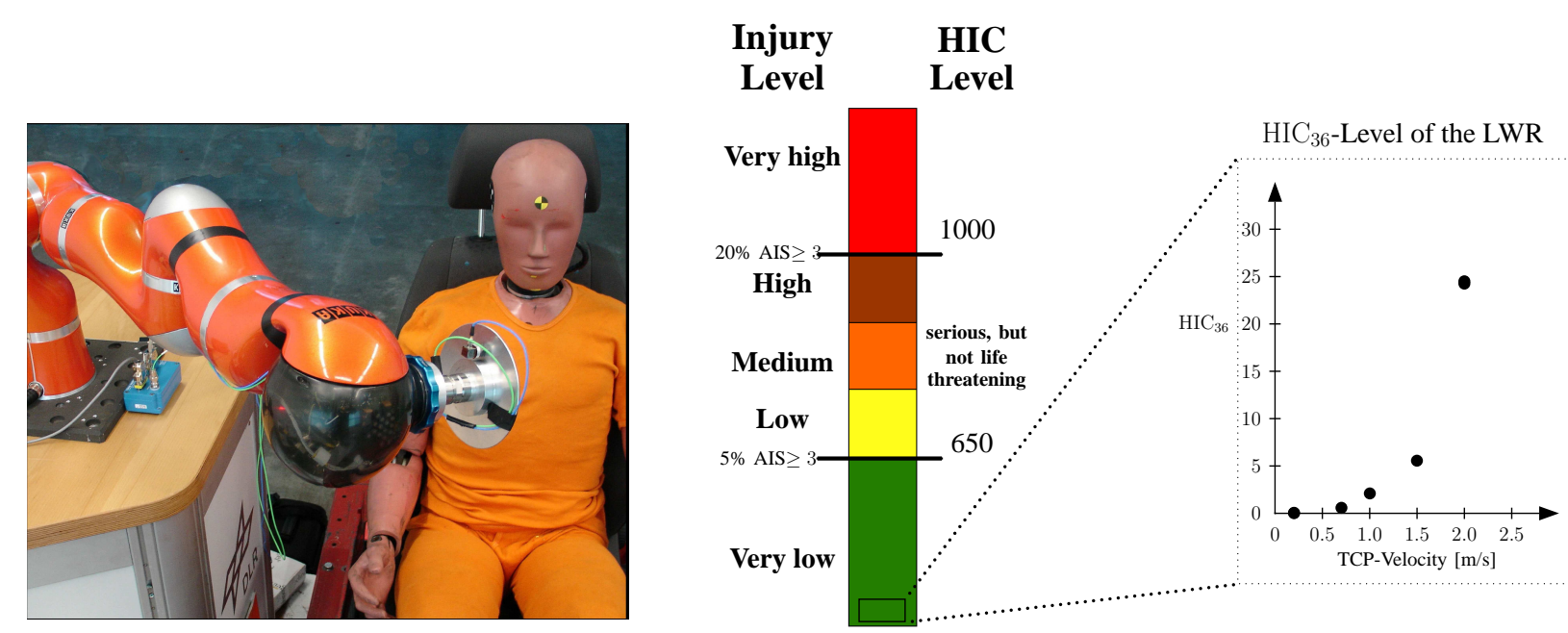

Fig. 6. Potential injury level of the human head caused by an impact of the DLR LWR expressed by the Head Injury Criterion (HIC).

implemented algorithm, both strategies are unified; deviation in specified directions and deceleration can be combined and arbitrarily mixed within the optimization problem.

\section{Safety Evaluation for Physical Human-Robot Interaction}

The desired coexistence of robotic systems and humans in the same physical domain, by sharing the same workspace and cooperating in a physical manner, poses the very fundamental problem of ensuring safety for the user and the robot. Joint torque sensing, together with a good robot model are used within the LWR software for fast detection of collision or failure.

In order to measure the potential danger emanating from the DLR LWR, impact tests at the Crash Test Center of the German Automobile Club ADAC (see Fig. 6 left) were conducted. The robot was commanded to move on predefined trajectories and hit various dummy body parts. It stopped either if the measured joint torque exceeded a maximal value, or if a collision was detected using an external torque observer. Inputs to the observer are the joint torques and motor positions [5]. To indicate the resulting level of injury, so-called Severity Indices were evaluated. We chose to present here the results of the Head Injury Criterion (HIC, introduced in [6]), but other indices for the head, neck, and chest were measured as well. The HIC evaluates the resulting head acceleration during an occuring impact. It is the most prominent and widely used measure to quantify the injury level of human beings caused by car accidents and was introduced to robotics in [7], [8].

In our evaluation we concentrated on unexpected impacts of a smooth surface on the three observable body regions head, neck, and chest. Injury mechanisms caused by sharp tools or similar injury sources 
were not taken into consideration since these mechanisms cannot be measured with standard crash-test dummies.

In Fig. 6 the resulting HIC values are plotted with respect to the impact velocity of the robot. The corresponding injury level is defined by the Euro $\operatorname{NCAP}^{2}$ [9]. It is subdivided into five categories from very low (green) to very high (red). In order to classify an impact into the green labeled region, the occuring HIC must not exceed 650. This particular value corresponds to a resulting probability of serious injury of $5 \%$.

As indicated in Fig. 6, the HIC caused by the LWR is below 25 at maximum velocity of $2 \mathrm{~m} / \mathrm{s}$ which corresponds to a very low injury level. Furthermore, all additionally evaluated severity measures ranged within the lowest quarter of the green indicated area as well, i.e. the potential danger emanating from the LWR is intrinsically very low with respect to the investigated mechanisms of injury. These are surprising and gratifying results. To our knowledge, they represent the first systematic experimental evaluation of possible injuries during robot-human crashes using standardized testing facilities.

\section{Automatic Planning of Robust Assembly Applications Using Impedance Control}

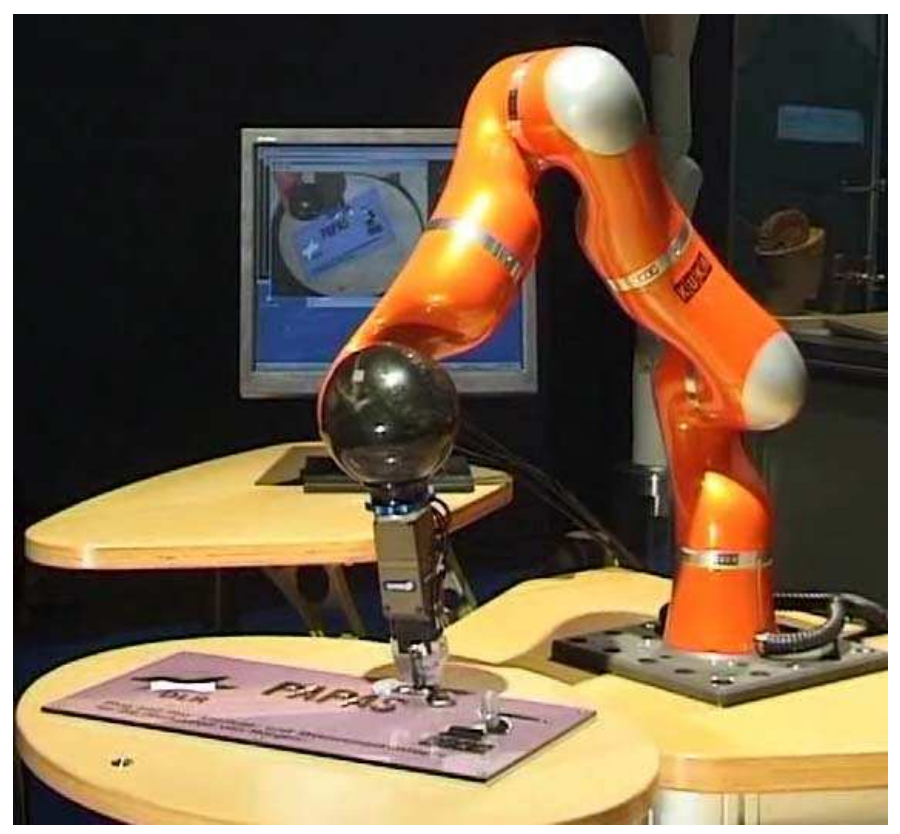

Fig. 7. Demonstrating robust assembly at the Automatica fair.

A typical example for the usage of the described Cartesian impedance control in an industrial environment is robotic assembly. This is a demanding task because two or more parts have to be brought into contact and aligned properly by the robot. Uncertainties due to positioning errors of the robot or inexact geometrical models are inevitable and have to be taken into account. If high accuracy is needed, costs for the setup of such a work cell explode. Especially the installation of precise part feeding mechanisms, high-resolution sensor systems, custom made grippers with passive compliance and the tedious programming of the robot are expensive.

Conventional position-controlled industrial robots generally have difficulties in keeping two parts in stable contact. Model uncertainties and lack of proper force feedback may lead to high reaction forces which could damage the parts, the tools, or the robot. One common solution is the use of an additional force/torque-sensor at the wrist for force control, but due to the high mass and stiffness of an industrial robot, the velocity has to be lowered dramatically to ensure stability. If high execution speed is

\footnotetext{
${ }^{2}$ The EuroNCAP is designed to provide a fair, meaningful and objective assessment of the impact performance of cars.
} 
desired, special (passively) compliant tools can be used instead to limit the contact forces. For assembly tasks, industrial robots are usually equipped with a so-called Remote Center Compliances [10], whose properties (center of compliance and stiffness parameters) are defined by the mechanical setup and therefore changeable by hardware modification only. For optimal performance, a different RCC would be needed for every type of part to be assembled. The impedance controlled DLR LWR combines the advantages of both systems. The passivity based controller allows stable contact even for high velocities due to the included joint torque feedback. Additionally, the active implementation of compliant behavior has the advantage that the parameters are adjustable by software and can be adapted individually for every subtask.

After getting into contact, proper alignment of the parts despite the inevitable uncertainties is the most challenging part of an assembly task. Usually this requires tedious and expensive hand-optimization of the trajectories for every type of object. In order to simplify this procedure, we developed an algorithm, which allows automatic planning of robust assembly applications. The algorithm takes the part geometries and information about the expected uncertainties as input and generates a parameterized robot program for the robust assembly of the parts.
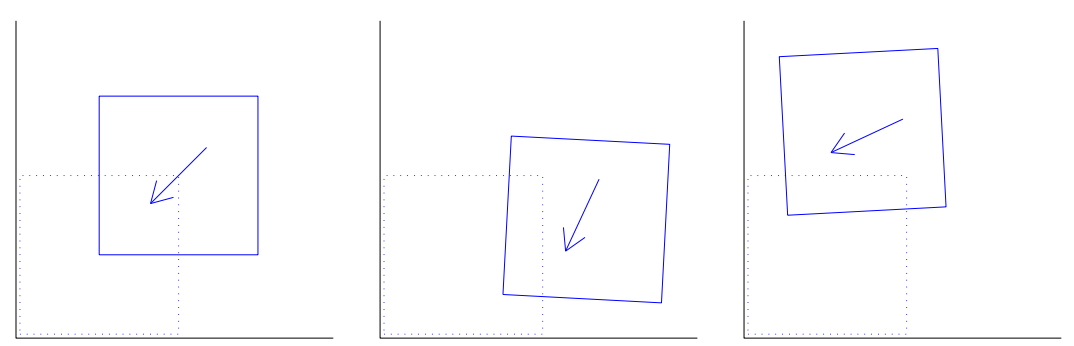

Fig. 8. Basic principle of the alignment of parts by pushing: assuming that the starting position and the pushing direction is within certain bounds, the square will always end up perfectly aligned with the corner (dotted position).

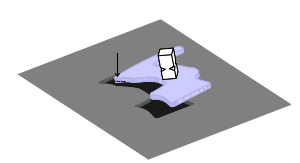

(a) immerse first vertex

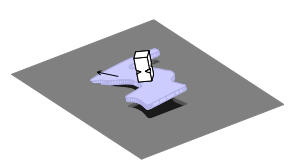

(b) align vertex

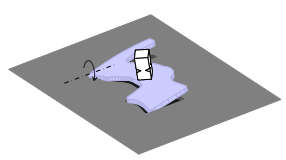

(c) immerse second vertex

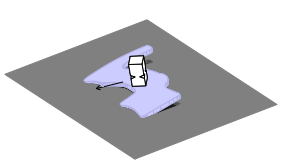

(d) align whole part (e) straighten up part along edge

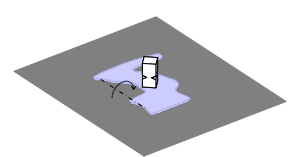

Fig. 9. Basic assembly strategy for an example part.

The basic principle of the algorithm is based on the observation that it is very easy to align a square with a rectangular corner (see Fig. 8): the square is simply pushed in the direction of the corner. Using this method, the contact forces implicitly help to align the parts. To succeed, the starting position and the exact direction of the movement are irrelevant as long as they are located within certain regions. These regions of attraction (ROA) are therefore the key elements for robust assembly: instead of positioning the part with high accuracy at the desired position, it is enough if the robot is able to position the part within the ROA and push it compliantly in the calculated direction. For a given part shape, our algorithm is able to calculate the ROAs for all contact points and for all pushing directions. The motion providing the maximal ROA and thus maximal robustness is selected for execution. Usually, one contact point is not enough for aligning the part properly, but it is not difficult to generalize the idea and use a pair or a sequence of contact points instead. Details about the algorithm can be found in [11]. At the current state the algorithm is only able to handle planar geometries. This is enough for flat parts whose height is small compared to its lateral dimensions. An example assembly for this case can be seen in Fig. 9. It takes advantage of the ROA by slightly tilting the part before insertion. The extension of the algorithm to real 3D parts is in progress. 
The test setup in our lab consists of three planar example parts with a clearance of less than $0.1 \mathrm{~mm}$. The DLR lightweight robot fetches the parts from a magazine and inserts them into the appropriate holes of a puzzle plate (Fig. 7). In order to evaluate the robustness statistically, numerous experiments with artificial uncertainty were accomplished. The randomized uncertainty (in the range of 0 to $20 \mathrm{~mm}$ lateral and 0 to $\pm 5^{\circ}$ rotational) was added to the known position of the plate. A graphical evalution for the part shown in Fig. 9 is given in Fig. 10. Within the marked area (up to $5 \mathrm{~mm}$ lateral and up to $2^{\circ}$ rotational offset), the method provided $100 \%$ success rate for all parts. An extended version of the experiment was presented at the Automatica 2006 fair in Munich. The puzzle plate was movable and its position was determined by a low-cost vision system. The position uncertainties of the whole system (robot and vision system) were up to $3 \mathrm{~mm}$ for translations and $2^{\circ}$ for rotations. As expected from our previous results, the experiment performed well throughout the fair.

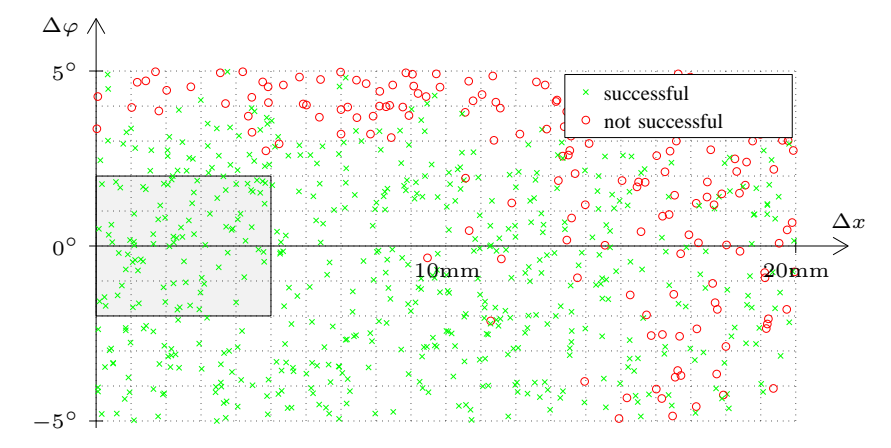

Fig. 10. The experimental results of the compliance based assembly for the considered range of position estimation errors. Each mark represents one insertion try. The assembly was carried out 751 times with an overall success rate of 558 (74.3\%). Within the marked area, all 88 tries were successful, which shows the robustness provided by the optimized ROA. However, the asymmetry of the results indicates that the trajectory generation based on the selected ROA can still be improved.

\section{Justin: A “Soft” Humanoid with Lightweight TeChnology}

Based on the DLR lightweight robots a humanoid upper-body-system Justin was set up as a testbed for studying two-handed manipulation ([12], Fig. 11). This system consists of two four-fingered DLRhands-II [13] and two 7DOF lightweight robots mounted on a 3DOF movable torso. Utilizing the modular structure of the arms and hands, the system has been assembled symmetrically in a humanoid configuration with a right-handed and a left-handed sub-system. Furthermore, the technologies developed for the DLR arms have also been utilized in the design of the torso. Consequently, all 41DOF of the torso, the arms, and the hands have joint torque sensors in addition to the common motor position sensors. This facilitates the implementation of coordinated control algorithms for Justin, since the same control concepts can be used for all the joints.

For two-handed manipulation tasks we use passivity based controllers which are derived from the Cartesian impedance control concepts developed for the arms. Inner loop joint torque controllers are used for all joints in order to overcome the negative effects of high motor inertia and friction due to the gears. The set-points (desired position) for these underlying torque controllers are determined by appropriate impedance behaviors, taking account of the peculiarities in coordinated two-handed manipulation (Fig. 12).

Based on the position of the fingertips a virtual frame is defined for each hand. The fingertips are connected via virtual (one-dimensional) interconnection springs to these virtual frames. By changing the rest lengths of the interconnection springs one can control the grasping forces of the right and the left hand [14]. In addition to these interconnection springs for grasping, two spatial springs define the motion of the arms. First, a coupling spring in between the arms is used to regulate the relative stiffness and the relative pose between the right and the left arm. Secondly, a virtual object spring is 
used to control the common motion of the arms. When the hands have grasped an object, one thus can move this object intuitively by moving the equilibrium pose of the object spring. The two spatial springs can easily be combined with the interconnection springs for the hands, by attaching the springs to the virtual hand frames rather than to the end-effector frames of the arms. By mapping the forces of all these virtual (visco-elastic) springs to the joints, one can generate whole-body motions in which the motions of the torso, the arms, and the hands are controlled in a coordinated way. However, these virtual springs clearly define only the Cartesian motion of the system, while the nullspace motion, i.e. the posture of the humanoid manipulator, must be controlled separately. For this we use additional impedance behaviors, which are mapped onto the robots nullspace by appropriate projection matrices. As nullspace impedances we currently use a joint limit avoidance criteria as well as the optimization of simple performance criteria. The applications for which this type of impedance has been used up to now include the coordinated transport of an object by two arms and hands, and the opening of a can by unscrewing the cap.

One interesting aspect in the performance of this control law for Justin is the distribution of the object motion to the joint motion of the fingers and the arms. In Fig. 13 the Euclidean norms of the joint velocities of the fingers and of the arms are shown for a step response according to the screwing motion. Here one can see that the velocity of the finger motion is considerably higher (c.f. the scaling in Fig. 13) than the arm motion. Furthermore, one can see that the fast part of the finger motion is finished much earlier than the arm motion [15].

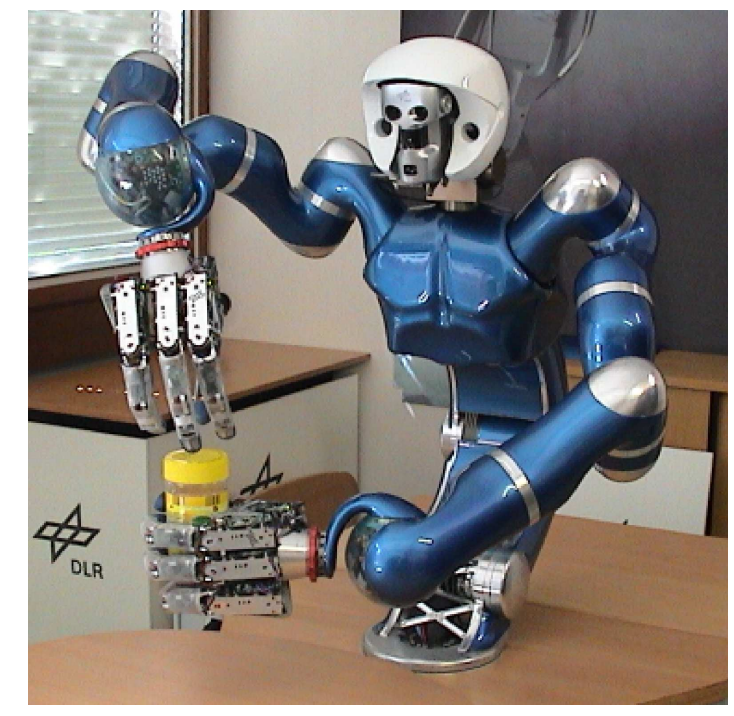

Fig. 11. The DLR humanoid manipulator Justin unscrewing a can.

\section{SUMMARY}

The DLR lightweight robots presented in this paper are excellent research platforms for experimentation of advanced robotics algorithms. Sensor technology, like the integrated joint torque sensors and link side potentiometers in addition to the common motor position sensors, allow for the implementation of safety features which go far beyond the state of the art in industrial robotics and facilitate the opening of new markets like medical applications or future service robotics scenarios. Potential industrial application fields are the fast automatic assembly as well as manufacturing activities performed in cooperation with humans (industrial robot assistant). The LWR technology was transferred to KUKA Roboter GmbH, which will bring the first arms on the market in close future.

The passivity of the joint level controllers and the Cartesian impedance controllers designed for these robots implies advantageous robustness properties. This is particularly relevant for applications in 


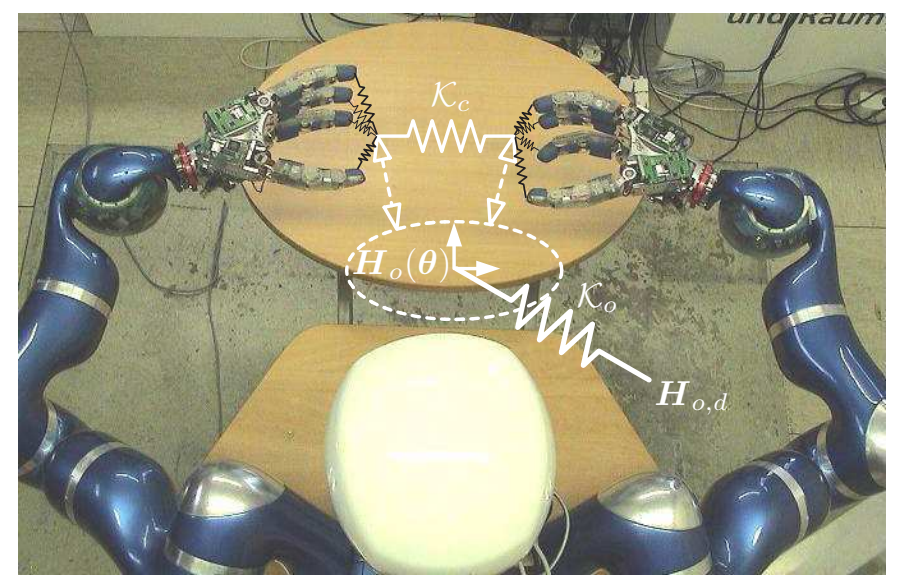

Fig. 12. Two-hand impedance behavior based on combination of object-level impedances of the hands (black) and arms (white).

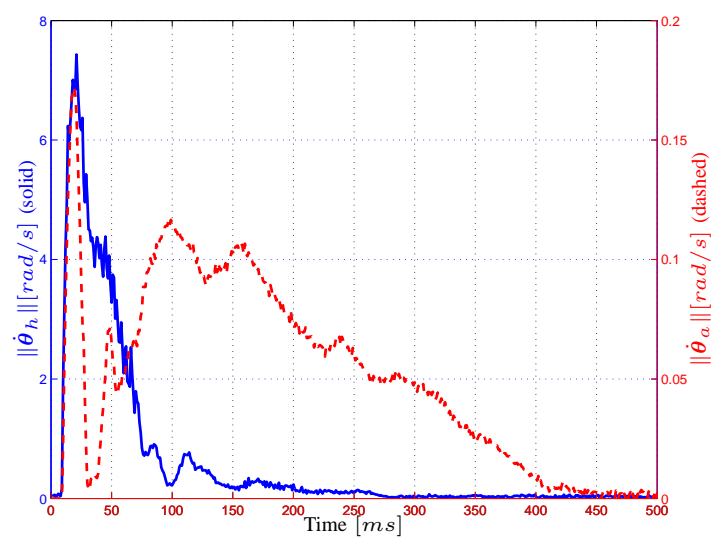

Fig. 13. Euclidean norm of the joint velocities of the arm $\left\|\dot{\boldsymbol{\theta}}_{a}\right\|$ (dashed) and the hand $\left\|\dot{\boldsymbol{\theta}}_{h}\right\|$ (solid) during a rotation about $0.3 \mathrm{rad}$.

which the model about the robot's environment is uncertain or only partly known. Impact experiments in cooperation with a German crash test center revealed that the potential danger (measured by the HIC, a widely used injury criterion in robotics) emanating from the lightweight arms intrinsically is very low. These results back up the expectation that these arms are very well suited for applications in which the robot must work close to humans.

The new control concepts also require new approaches in the planning and programming of tasks compared to industrial robots in order to increase the feasibility and robustness of complex assembly and service robotics applications. This is a topic of current research.

In two applications, the automated assembly and the two-handed manipulation with a humanoid manipulator, possible scenarios for the use of the DLR lightweight arms have been exemplified.

\section{REFERENCES}

[1] A. Albu-Schäffer, Ch. Ott, and G. Hirzinger, "A unified passivity based control framework for position, torque and impedance control of flexible joint robots," International Journal of Robotics Research, vol. 26, no. 1, pp. 23-39, 2007.

[2] _ _ "A passivity based cartesian impedance controller for flexible joint robots - Part II: Full state feedback, impedance design and experiments," in IEEE International Conference on Robotics and Automation, 2004, pp. 2666-2673.

[3] Ch. Ott, A. Albu-Schäffer, and G. Hirzinger, "A passivity based cartesian impedance controller for flexible joint robots - Part I: Torque feedback and gravity compensation," in IEEE International Conference on Robotics and Automation, 2004, pp. 2659-2665.

[4] G. Grunwald, G. Schreiber, A. Albu-Schäffer, and G. Hirzinger, "Programming by touch: The different way of human-robot interaction," IEEE Transactions on Industrial Electronics, vol. 50 No.4, pp. 659- 666, 2003.

[5] A. De Luca, A. Albu-Schäffer, S. Haddadin, and G. Hirzinger, "Collision detection and safe reaction with the DLR-III lightweight manipulator arm," in IEEE/RSJ International Conference on Intelligent Robots and Systems, 2006, pp. 1623-1630. 
[6] J. Versace, "A review of the severity index," Proc. 15th Stapp Conference, vol. SAE Paper No.710881, pp. 771-796, 1971.

[7] M. Zinn, O. Khatib, B. Roth, and J. Salisbury, "Playing it safe - human-friendly robots," IEEE Robotics and Automation Magazine, vol. 11, pp. 12-21, 2002.

[8] A. Bicchi and G. Tonietti, "Fast and soft arm tactics: Dealing with the safety-performance trade-off in robot arms design and control," IEEE Robotics and Automation Magazine, vol. 11, pp. 22-33, 2004.

[9] EuroNCAP, "European protocol new assessment programme - assessment protocol and biomechanical limits," 2003.

[10] D. E. Whitney and J. L. Nevins, "What is the remote center compliance and what can it do?" in 9th International Symposium on Industrial Robots, Washington, D. C., 1978.

[11] A. Stemmer, A. Albu-Schäffer, and G. Hirzinger, "An analytical method for the planning of robust assembly tasks of complex shaped planar parts," accepted for publication on ICRA 2007.

[12] Ch. Ott, O. Eiberger, W. Friedl, B. Bäuml, U. Hillenbrand, Ch. Borst, A. Albu-Schäffer, B. Brunner, H. Hirschmüller, S. Kielhöfer, R. Konietschke, M. Suppa, T. Wimböck, F. Zacharias, and G. Hirzinger, "A humanoid two-arm system for dexterous manipulation," in IEEE-RAS International Conference on Humanoid Robots, 2006.

[13] J. Butterfaß, M. Grebenstein, H. Liu, and G. Hirzinger, "DLR-Hand II: Next generation of a dextrous robot hand," in IEEE International Conference on Robotics and Automation, 2001, pp. 109-114.

[14] T. Wimböck, Ch. Ott, and G. Hirzinger, "Passivity-based object-level impedance control for a multifingered hand," in IEEE/RSJ International Conference on Intelligent Robots and Systems, 2006, pp. 4621-4627.

[15] — _ "Impedance behaviors for two-handed manipulation: Design and experiments," accepted for publication on ICRA 2007. 